\title{
Drag-Free Motion Control of Satellite for High-Precision Gravity Field Mapping
}

Ziegler, Bent Lindvig; Blanke, Mogens; Ziegler, Bent Lindvig; Blanke, Mogens

Published in:

Proceedings of the IEEE Conference on Control Applications, CCA' 2002

Link to article, DOI:

10.1109/CCA.2002.1040201

Publication date:

2002

Document Version

Publisher's PDF, also known as Version of record

Link back to DTU Orbit

Citation (APA):

Ziegler, B. L., Blanke, M., Ziegler, B. L. (Ed.), \& Blanke, M. (Ed.) (2002). Drag-Free Motion Control of Satellite for High-Precision Gravity Field Mapping. In Proceedings of the IEEE Conference on Control Applications, CCA' 2002 IEEE Press. https://doi.org/10.1109/CCA.2002.1040201

\section{General rights}

Copyright and moral rights for the publications made accessible in the public portal are retained by the authors and/or other copyright owners and it is a condition of accessing publications that users recognise and abide by the legal requirements associated with these rights.

- Users may download and print one copy of any publication from the public portal for the purpose of private study or research.

- You may not further distribute the material or use it for any profit-making activity or commercial gain

- You may freely distribute the URL identifying the publication in the public portal 


\title{
Drag-Free Motion Control of Satellite for High-Precision Gravity Field Mapping
}

\author{
Bent Ziegler, Mogens Blanke \\ Technical University of Denmark, Ørsted·DTU, Automation, Building 326, DK-2800 Lyngby, Denmark \\ Tel: +45 4525 3547, +45 4525 3565, Fax: +45 4588 1295, e-mail: blz@oersted.dtu.dk, mb@oersted.dtu.dk
}

\begin{abstract}
High precision mapping of the geoid and the Earth's gravity field are of importance to a wide range of ongoing studies in areas like ocean circulation, solid Earth physics and ice sheet dynamics. Using a satellite in orbit around the Earth gives the opportunity to map the Earth's gravity field in 3 dimensions with much better accuracy and spatial resolution than ever accomplished. To reach the desired quality of measurements, the satellite must fly in a low Earth orbit where disturbances from atmospheric drag and the Earth's magnetic field will perturb the satellite's motion. These effects will compromise measurement accuracy, unless they are accurately compensated by onboard thrusters. The paper concerns the design of a control system to performing such delicate drag compensation. A six degrees-of-freedom model for the satellite is developed with the model including dynamics of the satellite, sensors, actuators and environmental disturbances to the required micro-Newton accuracy. A control system is designed to compensate the non-gravitational disturbances on the satellite in three axes using an $\mathcal{H}_{\infty}$ design. Performance is validated against mission requirements.
\end{abstract}

Keywords: Spacecraft Attitude and Orbit Control, Drag Compensation, Drag-free motion.

\section{Introduction}

High precision gravity field mapping promise new insight in solid Earth physics, oceanography, ice sheet dynamics, geodesy and observation of possible sea level changes caused by global warming. The European Space Agency has planned a satellite dedicated for this purpose: Gravity field and Ocean Circulation Explorer (GOCE). The mission depends on accurate compensation of drag effects, referred to as drag-free motion control. The quality of the measurements are expected to yield results with high accuracy: $4 \mathrm{mE}$ for 3-dimensional rate of gravity and $1 \mathrm{~cm}$ for the geoid with a spatial resolution of $100 \mathrm{~km}$. The GOCE satellite is expected to be launched in 2006 [2].
Assessment of small anomalies in the gravity field is accomplished using a three-axis gradiometer. Differences in acceleration of the satellite and electrostatic suspended masses within the gradiometer are caused by disturbances on the satellite from external forces like air drag, and this must be compensated for.

The objective of this study has been the design of a control system that suppresses disturbances from environment well enough to meet the scientific requirements for the mission. This shall be done in the presence of disturbances from actuators and measurement noise. Requirements are given in terms of specifications for rotational and translational accelerations of the satellite. The actuator system used for all 6 degrees of freedom is a combination of an ion thruster and eight cold gas thrusters.

The paper gives a short introduction to the mission and presents performance specifications for the control system. The satellite is modelled with measurementand actuator systems. The essentials of main disturbances are analysed and the problem is formulated as an $\mathcal{H}_{\infty}$ control problem, using the requirements as performance and sensitivity specifications. A control system for drag compensation is designed, and controller performance is discussed [1].

\section{Satellite Orbit and Performance Requirements}

The orbit chosen for this mission is a near-polar sun synchronous circular low Earth orbit with an altitude of $250 \mathrm{~km}$ and an inclination $i$ of $96.5^{\circ}$.

The low orbit increase influence from gravity anomalies on the satellite motion but also cause an increment in the disturbance from the environment. In order to be able to accomplish scientific measurements of the desired quality the satellite accelerations should be kept below the specifications in Table 1 and Table 2 [1]. 
Table 1: Performance Specifications for Rotational Acceleration. Units $\left[\mathrm{rad} / \mathrm{s}^{2} / \sqrt{\mathrm{Hz}}\right]$

\begin{tabular}{|c|c|c|c|}
\hline Parameter & $\mathrm{f}<5 \mathrm{mHz}$ & $\mathrm{MBW}$ & $\mathrm{f}>100 \mathrm{mHz}$ \\
\hline$d^{2} \phi / d t^{2}$ & $1 \cdot 10^{-6}$ & $2.4 \cdot 10^{-8}$ & $1 \cdot 10^{-6}$ \\
$d^{2} \theta / d t^{2}$ & $1 \cdot 10^{-6}$ & $2.4 \cdot 10^{-8}$ & $1 \cdot 10^{-6}$ \\
$d^{2} \psi / d t^{2}$ & $1 \cdot 10^{-6}$ & $2.4 \cdot 10^{-8}$ & $1 \cdot 10^{-6}$ \\
\hline
\end{tabular}

Table 2: Performance Specifications for Translational Acceleration. Units $\left[\mathrm{m} / \mathrm{s}^{2} / \sqrt{\mathrm{Hz}}\right]$

\begin{tabular}{|c|c|c|c|}
\hline Parameter & $\mathrm{f}<5 \mathrm{mHz}$ & $\mathrm{MBW}$ & $\mathrm{f}>100 \mathrm{mHz}$ \\
\hline$d^{2} x / d t^{2}$ & $1 \cdot 10^{-7}$ & $2.5 \cdot 10^{-8}$ & $1 \cdot 10^{-6}$ \\
$d^{2} y / d t^{2}$ & $5 \cdot 10^{-7}$ & $2.5 \cdot 10^{-8}$ & $1 \cdot 10^{-6}$ \\
$d^{2} z / d t^{2}$ & $1 \cdot 10^{-7}$ & $2.5 \cdot 10^{-8}$ & $5 \cdot 10^{-7}$ \\
\hline
\end{tabular}

\section{Satellite Modelling}

The satellite has a long octagonal body with a small and symmetric cross section perpendicular to the flight direction. This design is chosen in order to reduce air drag and disturbances to the gradiometer. To secure atmospheric drag stability, a stabiliser is added to the satellite structure causing the center of pressure $(\mathrm{CoP})$ to be located aft of the center of mass (CoM).

The satellite will be considered as a rigid body rotating with an angular velocity of one revolution per orbit. The satellite $x$-axis will be pointing in the direction of flight; the $z$-axis towards nadir and $y$ will complete the orthogonal right hand system. At the chosen altitude the angular velocity about $y$ is $\omega_{y}=0.00117 \mathrm{rad} / \mathrm{s}$

The principal moments of inertia for the satellite are considered to coincide with the satellite body axes $x, y$ and $z$ so the inertia tensor becomes a diagonal matrix. Estimated physical parameters for the satellite can be found in Table 3 .

$$
I=\left[\begin{array}{ccc}
I_{x} & 0 & 0 \\
0 & I_{y} & 0 \\
0 & 0 & I_{z}
\end{array}\right]
$$

Expressions for the dynamic equations of motion for a rigid body in a rotating frame are

$$
\boldsymbol{I} \frac{\mathrm{d} \boldsymbol{\omega}}{\mathrm{d} t}=\boldsymbol{N}-\boldsymbol{\omega} \times(\boldsymbol{I} \boldsymbol{\omega})
$$

for rotational motion where $\boldsymbol{\omega}$ denotes angular velocity and $\boldsymbol{N}$ denotes the torque acting on the satellite. For translational motion

$$
\frac{\mathrm{d} v}{\mathrm{~d} t}=-2 \omega \times v+\frac{\boldsymbol{F}}{m}
$$

when small higher order terms are neglected. Here $v$ is translational velocity and $\boldsymbol{F}$ is force.
Table 3: Physical parameters for the satellite

\begin{tabular}{|l|c|c|c|}
\hline Parameter & Symbol & Value & Unit \\
\hline Satellite mass & $m$ & 808.7 & $\mathrm{~kg}$ \\
\hline Principal moments & $I_{x}$ & 97.1 & $\mathrm{Nms}^{2}$ \\
of inertia & $I_{y}$ & 966.9 & $\mathrm{Nms}^{2}$ \\
& $I_{z}$ & 993.5 & $\mathrm{Nms}^{2}$ \\
\hline Cross sectional & $S_{x}$ & 0.8 & $\mathrm{~m}^{2}$ \\
areas & $S_{y}$ & 10 & $\mathrm{~m}^{2}$ \\
& $S_{z}$ & 5 & $\mathrm{~m}^{2}$ \\
\hline Drag coefficient & $C_{d}$ & 4.1438 & \\
\hline Magnetic & $m_{x}$ & 4.60 & $\mathrm{Am}^{2}$ \\
moment vector & $m_{y}$ & -0.65 & $\mathrm{Am}^{2}$ \\
& $m_{z}$ & 1.85 & $\mathrm{Am}$ \\
\hline Center of pressure & $r_{C o P, x}^{x}$ & 0 & $\mathrm{~m}$ \\
position for & $r_{C o P, y}^{x}$ & 0 & $\mathrm{~m}$ \\
x-direction pressure & $r_{C o P, z}^{x}$ & 0 & $\mathrm{~m}$ \\
\hline Center of pressure & $r_{C o P, x}^{y}$ & -0.29 & $\mathrm{~m}$ \\
position for & $r_{C o P, y}^{y}$ & 0 & $\mathrm{~m}$ \\
y-direction pressure & $r_{C o P, z}^{y}$ & 0 & $\mathrm{~m}$ \\
\hline Center of pressure & $r_{C o P, x}^{z}$ & -0.49 & $\mathrm{~m}$ \\
position for & $r_{C o P, y}^{z}$ & 0 & $\mathrm{~m}$ \\
z-direction pressure & $r_{C o P, z}^{z}$ & 0 & $\mathrm{~m}$ \\
\hline
\end{tabular}

In order to model the spacecraft dynamics, it is necessary to add the influence from environmental disturbances to the dynamic equations of motion. For an orbit with an altitude of $250 \mathrm{~km}$, the atmospheric drag is the most important disturbance but also a model the influence from the Earth's magnetic field on the satellite will be included.

\subsection{Atmospheric drag}

The acceleration of a satellite in low earth orbit due to atmospheric drag can be written as [5]

$$
\gamma^{\prime}=-\frac{1}{2} \rho \frac{S C_{d}}{m} V_{a t m}^{\prime} V_{a t m}^{\prime}
$$

where $\rho$ is the atmospheric density, $S$ is the effective surface normal to the velocity vector, $m$ is the mass, $C_{d}$ is the drag coefficient of the body and $V_{a t m}^{\prime}$ is the velocity of the satellite relative to the atmosphere. Rewriting Eq. 4 gives the force acting on the satellite

$$
\boldsymbol{F}_{\text {aero }}^{\prime}=-\frac{1}{2} \rho S C_{d} V_{a t m}^{\prime} V_{a t m}^{\prime}
$$

The atmospheric density for an orbit at the altitude of $250 \mathrm{~km}$ is calculated using the atmosphere model MSISE-90 with input shown in Table 4. This yields the atmospheric density shown in Figure 1.

Table 4: Atmosphere density model parameters

\begin{tabular}{|c|c|}
\hline $\mathrm{F}_{10.7}$ (previous day) & $140 \cdot 10^{-22} \mathrm{Jm}^{-2}$ \\
\hline $\mathrm{F}_{10.7}(3$-month average) & $140 \cdot 10^{-22} \mathrm{Jm}^{-2}$ \\
\hline$A_{p}$ & $15 \mathrm{nT}$ \\
\hline
\end{tabular}




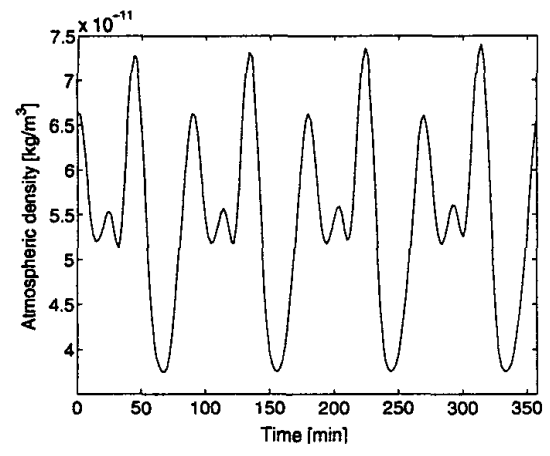

Figure 1: Atmospheric density through 4 orbits at 250 $\mathrm{km}$ altitude

The velocity relative to the atmosphere is a function of the absolute velocity of the satellite, $\boldsymbol{V}^{\prime}$, the angular velocity of the atmosphere, $\boldsymbol{\omega}_{\text {atm }}$, and the radius vector, $\boldsymbol{R}$, from the Earth center to the satellite.

$$
\boldsymbol{V}_{\text {atm }}^{\prime}=\boldsymbol{V}^{\prime}-\omega_{a t m} \times \boldsymbol{R}
$$

Assuming the angular velocity of the atmosphere as of the Earth and taking into account the inclination of the orbit, $i$, the velocity of the satellite relative to the atmosphere can be expressed as

$$
\begin{aligned}
V_{a t m}^{\prime} & =\left[\begin{array}{c}
V-R \omega_{\oplus} \cos i \\
R \omega_{\oplus} \sin i \cos \left(\omega_{0} t\right) \\
0
\end{array}\right] \\
V_{a t m}^{\prime} & \approx V-R \omega_{\oplus} \cos i
\end{aligned}
$$

where $\omega_{\oplus}$ is the angular velocity of the Earth about its axis [5]. The speed of the spacecraft, $V$, is derived from the gravitation constant of Earth, $\mu_{\oplus}$, and the distance from the geocenter to the spacecraft as

$$
V=\sqrt{\frac{\mu_{\oplus}}{R}}=\sqrt{\frac{3.9860 \cdot 10^{14} \mathrm{~m}^{3} / \mathrm{s}^{2}}{6.6281 \cdot 10^{6} \mathrm{~m}}}=7.7548 \frac{\mathrm{km}}{\mathrm{s}}
$$

The approximation in Eq. 8 follows from assuming

$$
V-R \omega_{\oplus} \cos i \gg R \omega_{\oplus} \sin i \cos \left(\omega_{0} t\right)
$$

Angular errors should be taken into account when finding the force acting on the satellite. This is done by applying the following rotation matrix to $V_{a t m}^{\prime}$

$$
\boldsymbol{A}=\left[\begin{array}{ccc}
1 & \psi & -\theta \\
-\psi & 1 & \phi \\
\theta & -\phi & 1
\end{array}\right]
$$

where rotations are $\phi$ about $x, \theta$ about $y$ and $\psi$ about $z$. The force acting on the satellite is then

$$
\boldsymbol{F}_{\text {aero }}=-\frac{1}{2} \rho S C_{d} V_{\text {atm }}^{\prime} \boldsymbol{A} \boldsymbol{V}_{\text {atm }}^{\prime}
$$

Because the CoP is located at a distance to CoM, this will cause a torque on the spacecraft

$$
N_{\text {aero }}=\boldsymbol{r}_{\text {CoP }} \times F_{\text {aero }}
$$

where $r_{C o P}$ is the vector from $\mathrm{CoM}$ to $\mathrm{CoP}$ (see Table 3). The CoP position differs for forces acting on different spacecraft axes. Hence

$$
\begin{aligned}
N_{\text {aero }} & =\boldsymbol{r}_{\text {CoP }}^{x} \times\left[\begin{array}{c}
F_{\text {aero, } x} \\
0 \\
0
\end{array}\right]+\boldsymbol{r}_{\text {CoP }}^{y} \times\left[\begin{array}{c}
0 \\
F_{\text {aero }, y} \\
0
\end{array}\right] \\
& +\boldsymbol{r}_{C o P}^{z} \times\left[\begin{array}{c}
0 \\
0 \\
F_{\text {aero }, z}
\end{array}\right]
\end{aligned}
$$

Due to the position of the CoP's relative to the CoM, the atmospheric drag causes a restoring torque about the spacecraft axes $y$ and $z$.

\subsection{The Earth's Magnetic Field}

Interaction between a magnetic moment in the spacecraft and the Earth's magnetic field causes a torque on the spacecraft

$$
N_{B}=m_{B} \times B
$$

$m_{B}$ is the magnetic moment of the spacecraft and $B$ is the Earth's magnetic field intensity at the spacecraft's position. Different models exist for the Earth magnetic field and a tilted dipole model, deviating from the $\boldsymbol{Z}_{\oplus^{-}}$ direction by $17^{\circ}$, has sufficient accuracy [4]. This model describes the components of the magnetic field as

$$
\boldsymbol{B}=\boldsymbol{A} \frac{m_{\oplus}}{R^{3}}\left[\begin{array}{c}
\cos \left(\omega_{0} t-\eta_{m}\right) \sin \left(\xi_{m}\right) \\
-\cos \left(\xi_{m}\right) \\
2 \sin \left(\omega_{0} t-\eta_{m}\right) \sin \left(\xi_{m}\right)
\end{array}\right]
$$

where

- $\boldsymbol{A}$ is the rotation matrix from Eq. 11

- $m_{\oplus}$ is the Earth magnetic dipole strength.

- $R$ is the distance from the center of the Earth.

- $\omega_{0} t$ is the angle from the ascending node to current spacecraft position.

- $\eta_{m}$ is a phase angle measured from the ascending node of the orbit relative to the geographic equator to the ascending node of the orbit relative to the geomagnetic equator.

- $\xi_{m}$ is the instantaneous inclination of the orbit plane to the geomagnetic equator.

Both $\eta_{m}$ and $\xi_{m}$ are time varying but as the orbit period for the spacecraft is much smaller than of the rotation of Earth, these variables are considered constant. The range for $\xi_{m}$ is $i \pm 17^{\circ}$ and, for a near polar orbit, the range for $\eta_{m}$ can be approximated to $\pm 17^{\circ}$.

The dynamic equations of motion Eq. 2 and Eq. 3 can now be written as

$$
I \frac{\mathrm{d} \boldsymbol{\omega}}{\mathrm{d} t}=-\boldsymbol{\omega} \times(\boldsymbol{I} \boldsymbol{\omega})+N_{\text {aero }}+\boldsymbol{N}_{B}+\boldsymbol{N}_{c}
$$




$$
\frac{\mathrm{d} v}{\mathrm{~d} t}=-2 \boldsymbol{\omega} \times \boldsymbol{v}+\frac{1}{m} \boldsymbol{F}_{\text {aero }}+\frac{1}{m} \boldsymbol{F}_{\mathrm{c}}
$$

where $\boldsymbol{N}_{c}$ and $\boldsymbol{F}_{c}$ denote torque and the force supplied by the propulsion system.

\subsection{Propulsion System}

The propulsion system used for applying torques and forces on the satellite consists of a combination of a single ion thruster and eight cold gas thrusters. The ion thruster compensates for atmospheric drag in the direction of flight $(x)$, which is the strongest perturbation on the satellite motion. The cold gas thrusters that control the remaining five degrees of freedom are placed as shown on Figure 2. The thrusters are numbered from 1 to 8 and their respective thrust vectors are shown as arrows on the figure. This combination of ion and cold gas thrusters yield the noise profiles shown in Figure 3.

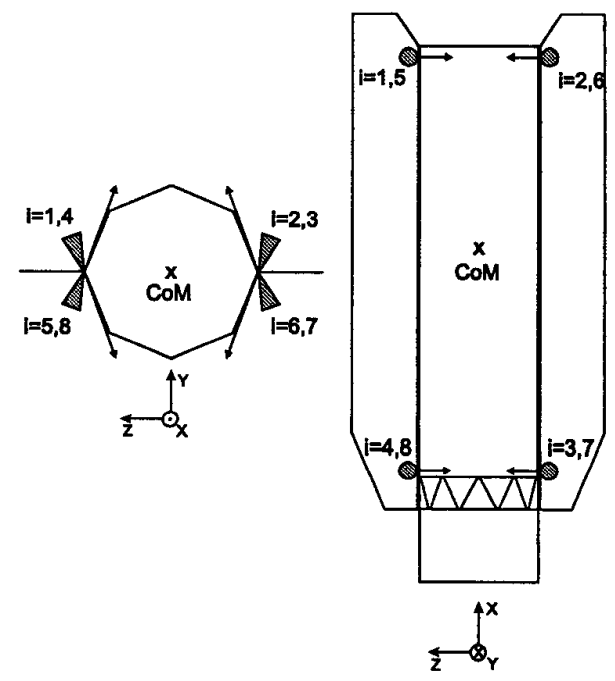

Figure 2: Cold gas thruster configuration

The thrusters show a second order behaviour with a bandwidth of $20 \mathrm{~Hz}$ and a damping coefficient of 0.7 . This gives the following relationship between commanded and obtained control signal about an axis $i$

$$
\begin{aligned}
\dot{x}_{t h} & =\left[\begin{array}{cc}
-176 & -15791 \\
1 & 0
\end{array}\right] \boldsymbol{x}_{t h}+\left[\begin{array}{c}
15791 \\
0
\end{array}\right]\left(u_{i, c}+v_{i}\right) \\
u_{i} & =\left[\begin{array}{ll}
0 & 1
\end{array}\right] \boldsymbol{x}_{t h}
\end{aligned}
$$

Here $u_{i, c}$ is the commanded control signal, $u_{i}$ is the obtained control signal and $v_{i}$ is the noise from the thrusters. The reason for the non-linearity at low frequencies in the noise profile for $F_{y}$ is that the ion thruster is canted by an angle of $2.8^{\circ}$ with respect to the $y$-axis to allow for a redundant ion thruster. Therefore a component of the ion thrust is directed towards $y$ influencing noise profile.
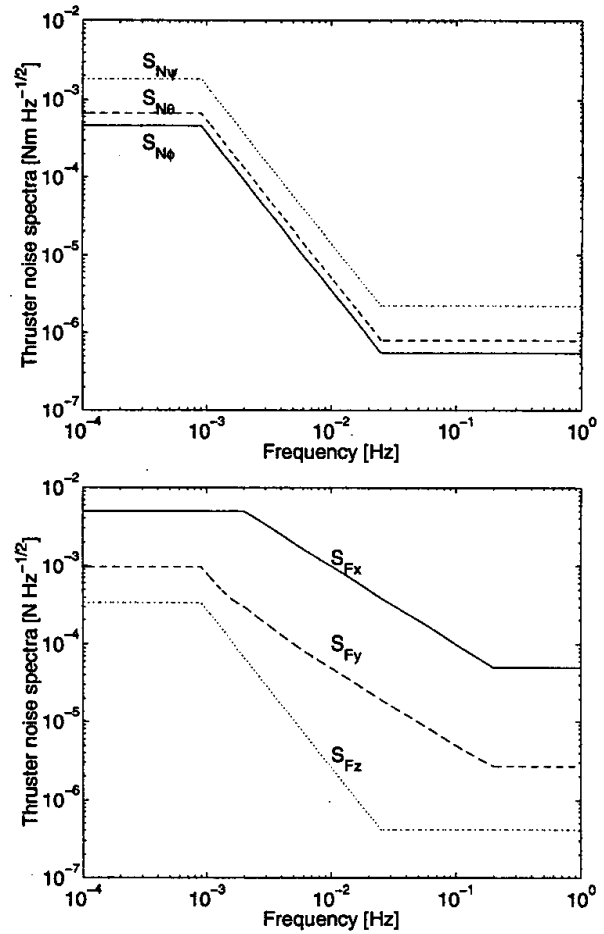

Figure 3: Noise profiles for torques and forces applied to the satellite

\subsection{Gradiometer}

For measuring satellite accelerations a gradiometer is used. This consists of six accelerometers in configuration that each can measure linear accelerations along three nominally orthogonal axes. From these measurements the satellite accelerations along 6 degrees of freedom are derived. The accelerometers are based on electrostatic suspension of proof masses adding second order dynamics to the measurements [1]. Measurement bandwidth is $1 \mathrm{~Hz}$, damping coefficient is 0.7 and the steady state gain is 1 . Gradiometer noise is considered as white noise with the following spectral densities for rotational and translational acceleration

$$
\begin{aligned}
S_{g m, r} & =3.78 \cdot 10^{-11} \frac{\mathrm{rad}}{s^{2} \sqrt{H z}} \\
S_{g m, t} & =3.78 \cdot 10^{-11} \frac{\mathrm{m}}{s^{2} \sqrt{H z}}
\end{aligned}
$$

Acceleration along any axis $i$ is thus measured as

$$
\begin{aligned}
\dot{x}_{g m} & =\left[\begin{array}{cc}
-8.8 & -39.5 \\
1 & 0
\end{array}\right] \boldsymbol{x}_{g m}+\left[\begin{array}{c}
39.5 \\
0
\end{array}\right] a_{i} \\
a_{i, m} & =\left[\begin{array}{ll}
0 & 1
\end{array}\right] \boldsymbol{x}_{g m}+w_{i}
\end{aligned}
$$

where $a_{i, m}$ is the measured acceleration, $a_{i}$ is the true acceleration and $w_{i}$ is the sensor noise with the spectral density from Eq. 20 or Eq. 21. 


\section{Control System Design}

A control system is designed that keeps the accelerations of the satellite below the performance requirements from Table 2 and Table 1 . For this purpose a set of continuous $\mathcal{H}_{\infty}$-controllers are used. Keeping in mind that the system probably should be discretised at a later point the bandwidth of the controllers will be considered a restriction of the controller design.

The dynamic equations for translational motion Eq. 18 reveal a cross-coupling from rotation of the spacecraft to translational motion. This cross-coupling is counteracted by a feed-forward term added to the control force $\boldsymbol{F}_{c}$ giving

$$
\begin{aligned}
\tilde{\boldsymbol{F}}_{c} & =\boldsymbol{F}_{c}-2 m(\boldsymbol{\omega} \times \boldsymbol{v}) \\
\frac{\mathrm{d} \boldsymbol{v}}{\mathrm{d} t} & =\frac{1}{m} \boldsymbol{F}_{\text {aero }}+\frac{1}{m} \tilde{\boldsymbol{F}}_{c}
\end{aligned}
$$

This enables the separation of control systems for rotational and translational motion.

Control system inputs will be based on raw measurements from the gradiometer. The noise from this device is much lower than the acceleration noise caused by the thrusters and the advantages of including a Kalman filter for state estimation are thus negligible.

Influence from atmospheric drag and magnetic field on satellite dynamics depend on the parameters $\rho, \omega_{0} t, \xi_{m}$ and $\eta_{m}$. The control system is therefore designed for the parameter combination where angular errors have the largest effect on satellite dynamics.

Table 5: Worst case parameter values

\begin{tabular}{|c|c|}
\hline Parameter & Value \\
\hline$\rho$ & $7.28 \cdot 10^{-11} \mathrm{~kg} / \mathrm{m}^{3}$ \\
$\omega_{0} t$ & $0 \mathrm{rad}$ \\
$\xi_{m}$ & $79.5^{\circ}$ \\
$\eta_{m}$ & $0^{\circ}$ \\
\hline
\end{tabular}

\subsection{Setup for Controller Design}

The gradiometer bandwidth of $1 \mathrm{~Hz}$ is only ten times higher than the upper limit of MBW so the gradiometer dynamics (Eq. 22) is included in the model. The thruster dynamics (Eq. 19) with a bandwidth of $20 \mathrm{~Hz}$ is neglected for controller synthesis but used for later validation.

The configuration for $\mathcal{H}_{\infty}$-controller synthesis is shown on Figure 4. This setup applies to controllers for both rotational and translational motion. $\boldsymbol{G}$ contains satellite dynamics, $\boldsymbol{G} \boldsymbol{m}$ contains the gradiometer, $\boldsymbol{K}$ is the controller and $W_{g m}, W_{t h}, W_{c}$ and $W_{p}$ are weight functions. Noise from measurements and propulsion system are represented by $w_{1}$ and $w_{2}$. The output $z_{1}$

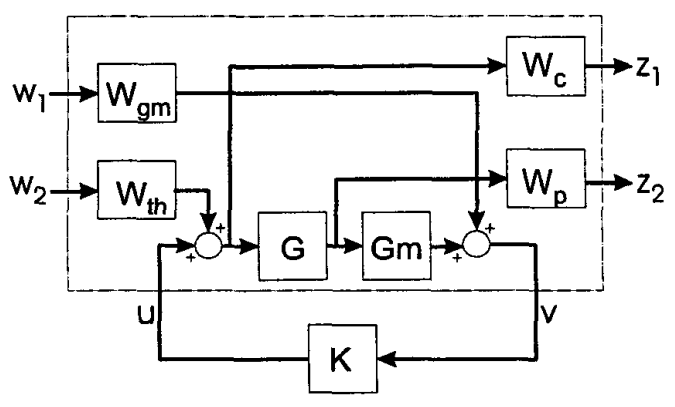

Figure 4: Setup used for control system synthesis

is the weighted force and torque supplied by the control system and $z_{2}$ is the weighted output for satellite acceleration. $v$ is measured acceleration and $u$ is control signal. In order to suppress acceleration noise at the desired frequencies it has proved sufficient only to include dynamics in the weight $W_{p}$ while $\boldsymbol{W}_{g m}, \boldsymbol{W}_{t h}$ and $\boldsymbol{W}_{c}$ are constant matrices.

The following three weight matrices are used for both control systems

$$
\begin{aligned}
\boldsymbol{W}_{g m} & =3.78 \cdot 10^{-11} \cdot I_{3 \times 3} \\
W_{c} & =1 \cdot 10^{-2} \cdot I_{3 \times 3} \\
\boldsymbol{W}_{p} & =\frac{0.1(s+10 \cdot 2 \pi)^{2}}{\left(s+20 \cdot 10^{-3} \cdot 2 \pi\right)^{2}} \cdot I_{3 \times 3}
\end{aligned}
$$

while the weights $\boldsymbol{W}_{t h}$ for rotational and translational motion are

$$
\begin{aligned}
& \boldsymbol{W}_{t h, r}=150\left[\begin{array}{ccc}
4.6 \cdot 10^{-4} & 0 & 0 \\
0 & 6.6 \cdot 10^{-4} & 0 \\
0 & 0 & 1.8 \cdot 10^{-3}
\end{array}\right] \\
& \boldsymbol{W}_{t h, t}=100\left[\begin{array}{ccc}
5 \cdot 10^{-3} & 0 & 0 \\
0 & 9.7 \cdot 10^{-4} & 0 \\
0 & 0 & 3.4 \cdot 10^{-4}
\end{array}\right]
\end{aligned}
$$

$\boldsymbol{W}_{g m}$ is chosen as the intensity of the gradiometer noise while the $\boldsymbol{W}_{t h}$ values are scaled according to the low frequency intensity of the noise from the propulsion system. $\boldsymbol{W}_{c}$ is chosen low to allow a certain noise on the control signal, while acceleration noise is suppressed heavily by the $\boldsymbol{W}_{p}$ function. This suppression is harder at lower frequencies where propulsion noise is strong but falls off when approaching the upper limit for the MBW.

\subsection{Control System Analysis}

Design using the setup above results in stabilising controllers for rotational and translational motion of 18 th and 14th order, respectively, before controller model reduction. The controller for rotational motion has natural modes of up to $35 \mathrm{rad} / \mathrm{s}$, while those for the translational control motion are below $25 \mathrm{rad} / \mathrm{s}$. These natural modes cause the minimum sampling frequency for the system to be $11.1 \mathrm{~Hz}$. 
To validate control system performance propulsion dynamics is included in the system. It is noted that this amendment does not affect system stability. Noise with the spectral profile shown in Figure 3 is then applied and the resulting acceleration noise plotted in Figure 5 against the requirements.
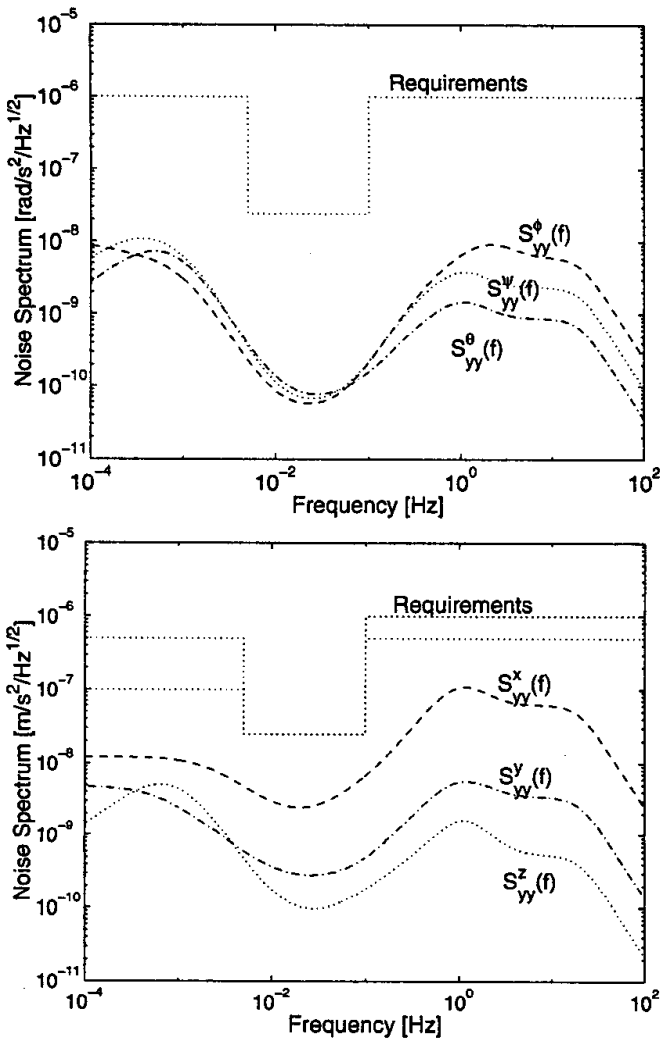

Figure 5: Acceleration spectra for rotational and translational motion

Requirements are seen to be met for both rotational and translational accelerations with comfortable margin. In rotational acceleration the spectra reach a maximum of $2 \%$ of the limit in the MBW. In translational accelerations the noise reaches $25 \%$ for $x$-axis acceleration at $0.1 \mathrm{~Hz}$, while accelerations along $y$ and $z$ reach their maximum of $2 \%$ of the limit at the same frequency.

If the requirements for noise rejection on any axis was to be tightened, a trade-off could be made in the design between noise suppression and control system bandwidth. A faster controller could keep the acceleration noise at a lower level by increasing the drop-off frequency but would also increase the minimum sampling frequency for the system. With the margins obtained, the high order of the controllers is easily reduced.

\section{Conclusions and Further Work}

A six-degree-of-freedom model for a satellite in low Earth orbit was derived. The model included propulsion, measurement systems, environmental disturbances from atmospheric drag and the Earth's magnetic field. The effects on satellite dynamics were included. The satellite itself was considered a rigid body. A continuous-time control system for drag compensation was designed for the satellite, treating the rotational and translational motions separately.

A setup for $\mathcal{H}_{\infty}$ controller synthesis was described and the designed controllers provided stability and sufficient performance for both control systems. The minimum margin was found for translational acceleration along the $\mathrm{x}$-axis where the noise spectrum reached 25 $\%$ of its upper limit [6].

\section{Acknowledgements}

The results described in this paper were mainly obtained during B. Ziegler's 6 months visit (stagiere) to the European Space Agency, ESA, at the Technology Centre (ESTEC) in the Netherlands. The support from ESA and the inspiration from Dr. M. Sallusti are gratefully appreciated.

\section{References}

[1] ESA, The Four Candidate Earth Explorer Core Missions - Gravity Field and Steady-State Ocean Circulations, ESA Publications Division, 1999.

[2] Website: www.esa.int/export/esaLP/goce.html, June 11th 2002.

[3] Wertz, J. R., editor, Spacecraft Attitude Determination and Control, D. Reidel Publishing Company, edition 6, 1991.

[4] Sidi, M. J., Spacecraft Dynamics \& Control, Cambridge University Press, 1997.

[5] Carrou, J., editor, Spaceflight Dynamics, Cepadues-Editions, 1st edition, 1995.

[6] Ziegler, B., Modelling and Control System Design for the ESA Satellite GOCE, Thesis at Technical University of Denmark, Ørsted-DTU, Automation, Lyngby, Denmark, 2001 